\title{
Interplay of Electrostatic and Hydrophobic Effects with Binding of Cationic Gemini Surfactants and a Conjugated Polyanion: Experimental and Molecular Modeling Studies
}

\author{
Hugh D. Burrows, ${ }^{*}, \uparrow$ María J. Tapia, ${ }^{*}$, Claudia L. Silva $^{\dagger}{ }^{\dagger}$ Alberto A. C. C. Pais, ${ }^{\dagger}$ \\ Sofia M. Fonseca, ${ }^{\dagger}$ João Pina, ${ }^{\dagger}$ J. Seixas de Melo, ${ }^{\dagger}$ Yujie Wang, ${ }^{\S}$ Eduardo F. Marques, ${ }^{\S}$ \\ Matti Knaapila," Andrew P. Monkman," Vasil M. Garamus, ${ }^{\perp}$ Swapna Pradhan, ${ }^{\text {and }}$ \\ Ullrich Scherf ${ }^{\#}$ \\ Departamento de Química and Faculdade de Farmácia, Universidade de Coimbra, \\ 3004-535 Coimbra, Portugal, Departamento de Química, Universidad de Burgos, Burgos 09001, Spain, \\ Departamento de Química, Universidade do Porto, 4169-007, Portugal, Department of Physics, University of \\ Durham, South Road, Durham DH1 3LE, United Kingdom, GKSS Research Centre, Max-Planck-Strasse 1, \\ D-21502 Geesthacht, Germany, and Makromolekulare Chemie, Bergische Universität Wuppertal, \\ D-42097 Wuppertal, Germany
}

Received: January 5, 2007; In Final Form: February 26, 2007

\begin{abstract}
Understanding factors responsible for the fluorescence behavior of conjugated polyelectrolytes and modulation of their behavior are important for their application as functional materials. The interaction between the anionic poly $\{1,4-$ phenylene-[9,9-bis(4-phenoxy-butylsulfonate)]fluorene-2,7-diyl\}copolymer (PBS-PFP) and cationic gemini surfactants $\alpha, \omega-\left(\mathrm{C}_{m} \mathrm{H}_{2 m+1} \mathrm{~N}^{+}\left(\mathrm{CH}_{3}\right)_{2}\right)_{2}\left(\mathrm{CH}_{2}\right)_{s}\left(\mathrm{Br}^{-}\right)_{2}(m-s-m ; m=12, s=2,3,5,6,10$, and 12) has been studied experimentally in aqueous solution. These surfactants are chosen to see whether molecular recognition and self-assembly occurs between the oppositely charged conjugated polyelectrolyte and gemini surfactant when the spacer length on the surfactant is similar to the intercharge separation on the polymer. Without surfactants, PBS-PFP exists as aggregates. These are broken up upon addition of gemini surfactants. However, as anticipated, the behavior strongly depends upon spacer length $(s)$. Fluorescence measurements show three surfactant concentration regimes: At low concentrations $\left(<2 \times 10^{-6} \mathrm{M}\right)$ quenching occurs and is most marked with the small spacer 12-2-12; at intermediate concentrations $\left(\sim 2 \times 10^{-6}-10^{-3} \mathrm{M}\right)$, fluorescence intensity is constant, with a 12-carbon spacer 12-12-12 showing the strongest fluorescence; above the critical micelle concentration $\left(\mathrm{CMC} ; \sim 10^{-3} \mathrm{M}\right.$ ) increases in emission intensity are seen in all cases and are largest with the intermediate spacers 12-5-12 and 12-6-12, where the spacer length most closely matches the distance between monomer units on the polymer. With longer spacer length surfactants, surface tension measurements for concentrations below the $\mathrm{CMC}$ reveal the presence of polymer-surfactant aggregates at the air-water interface, possibly reflecting increased hydrophobicity. Above the CMC, small-angle neutron scattering experiments for the 12-6-12 system show the presence of spherical aggregates, both for the pure surfactant and for polyelectrolyte/gemini mixtures. Molecular dynamics simulations help rationalize these observations and show that there is a very fine balance between electrostatic and hydrophobic interactions. With the shortest spacer 12-2-12, Coulombic interactions are dominant, while for the longest spacer 12-1212 the driving force involves hydrophobic interactions. Qualitatively, with the intermediate 12-5-12 and 126-12 systems, the optimum balance is observed between Coulombic and hydrophobic interactions, explaining their strong fluorescence enhancement.
\end{abstract}

\section{Introduction}

Molecular recognition through electrostatic, hydrophobic, or other interactions is of interest for both preparation of selfassembled supramolecular structures and advanced analytical applications. ${ }^{1,2}$ Conjugated polymers and polyelectrolytes, particularly those involving fluorene units, are now important materials, with applications including chemical and biological sensors, ${ }^{3-5}$ polymer light-emitting diodes, ${ }^{6-8}$ and photovoltaic

* Author to whom correspondence should be addressed. Fax: $(+351)$ 239 827703. E-mail: burrows@ci.uc.pt.

Universidade de Coimbra.

$\doteqdot$ Universidad de Burgos.

$\S$ Universidade do Porto.

"University of Durham.

${ }^{\perp}$ GKSS Research Centre.

\# Bergische Universität Wuppertal. cells. ${ }^{9}$ However, the fairly rigid fluorene-based $\pi$-conjugated polymers and copolymers tend to aggregate, which leads to low solubility, a marked decrease in the photoluminescence quantum yields, and a slight red shift in emission. ${ }^{10-12}$ Any strategy that breaks up these polymer aggregates and improves their photophysical (particularly the emission quantum yield) properties is of great interest from the point of view of applications of this class of materials. Among the strategies that have been attempted are the incorporation of bulky substituents, ${ }^{13}$ introduction of carbazole copolymer units, ${ }^{14}$ the interaction with surfactants, ${ }^{15-17}$ and most recently interaction with nanoparticle assemblies. ${ }^{18}$ An alternative explanation for the increase in the emission quantum yield of conjugated polymers upon interaction with surfactants is that it is not due to the disruption of polymer aggregates but is associated with changes in conformational 


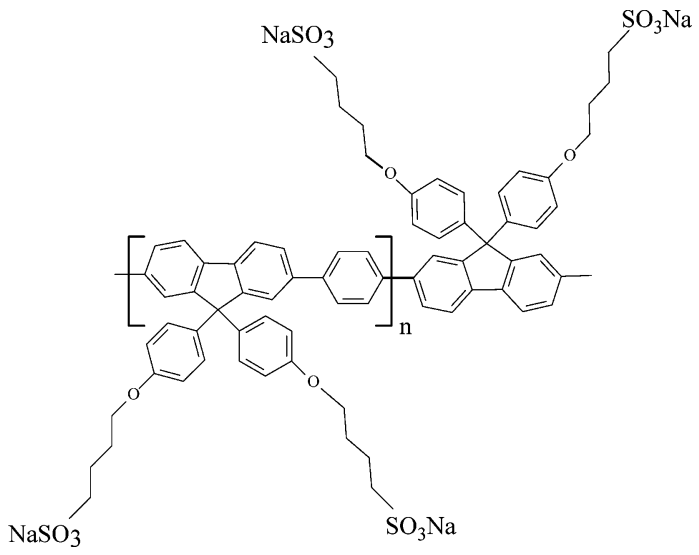

PBS-PFP

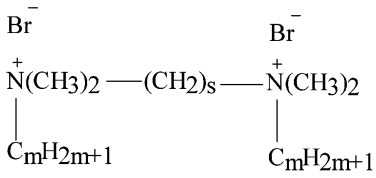

$\begin{array}{lc}\mathrm{m} & \mathrm{s} \\ 12 & 2,3,5,6,10,12 \\ 16,18 & 2\end{array}$

\section{$\mathrm{m}-\mathrm{s}-\mathrm{m}$}

Figure 1. Structure of poly\{1,4-phenylene-[9,9-bis(4-phenoxy-butylsulfonate)]fluorene-2,7-diyl (PBS-PFP) and gemini surfactants $\alpha, \omega$ $\left(\mathrm{C}_{m} \mathrm{H}_{2 m+1} \mathrm{~N}^{+}\left(\mathrm{CH}_{3}\right)_{2}\right)_{2}\left(\mathrm{CH}_{2}\right)_{s}\left(\mathrm{Br}^{-}\right)_{2}(m-s-m)$.

order. ${ }^{19-21}$ While it is possible that both factors are involved, the breakup of aggregates is likely to be a precursor of such conformational changes.

We have previously reported results on the interaction of the negatively charged polyfluorene poly $\{1,4$-phenylene-[9,9-bis(4-phenoxy-butylsulfonate)]fluorene-2,7-diyl\}copolymer, (PBSPFP, Figure 1) with surfactants, including the nonionic pentaethyleneglycol monododecyl ether $\left(\mathrm{C}_{12} \mathrm{E}_{5}\right),{ }^{22,23}$ anionic (sodium dodecyl sulfate), several cationic tetraalkylammonium surfactants with different structures (alkyl chain length, counterion, or double alkyl chain), ${ }^{24}$ and a cationic lecithin mimic. ${ }^{25}$ With the cationic tetralkylammonium bromides, where interactions are largely electrostatic, quenching of fluorescence, decrease in fluorescence lifetime, and formation of a new emission occurs at concentrations well below the critical micelle concentration due to polymer charge neutralization, which facilitates the formation of even larger polymer aggregates. With the anionic sodium dodecyl sulfate, fluorescence quenching is observed without the formation of any new emission. The most striking changes are observed in the presence of the nonionic pentaethyleneglycol monododecyl ether. In this case, where hydrophobic interactions are likely to play an important role, a blue shift in fluorescence emission and dramatic increases in lifetime and quantum yield are observed at surfactant concentrations in the region of the surfactant critical micelle concentration (CMC). This has been interpreted in terms of the breakup of PBS-PFP aggregates and the incorporation of one or more isolated chains of the polymer into long wormlike micelles. This is supported by small-angle neutron scattering (SANS) measurements ${ }^{17}$ and ${ }^{1} \mathrm{H} \mathrm{NMR}^{23}$ spectroscopy.

To broaden our understanding of the factors involved in these aggregation phenomena, it is interesting to choose a system where we can modulate the balance between electrostatic and hydrophobic interactions. For this, we chose to look at the interaction of PBS-PFP with gemini surfactants. These correspond to a relatively new generation of surfactants consisting of two hydrophobic chains and two head groups covalently attached by a spacer chain. ${ }^{26,27}$ Recently, much attention has been focused on their practical applications because they show very low CMCs compared with the corresponding monomeric surfactants, ${ }^{28}$ apart from having increased surface activity. ${ }^{26}$ In addition, within the context of molecular recognition and selfassembly,,$^{1,2}$ the gemini surfactants have two charges separated by a spacer, and by matching the distance between these charges to the separation between monomers in oppositely charged conjugated polyelectrolytes it may be possible to produce interesting functional materials, whose optical and photophysical properties are controlled by the balance between spacer length, Coulombic interactions, and hydrophobic effects.

The cationic gemini surfactants used in this work $\alpha, \omega$ $\left(\mathrm{C}_{m} \mathrm{H}_{2 m+1} \mathrm{~N}^{+}\left(\mathrm{CH}_{3}\right)_{2}\right)_{2}\left(\mathrm{CH}_{2}\right)_{s}\left(\mathrm{Br}^{-}\right)_{2}$ are referred to as $m-s-m$ (Figure 1), where $m$ and $s$ are the number of carbons of the tails and spacer alkyl chains, respectively. The spacer length has a pronounced effect on the shape of any aggregate formed. In the case of $12-s-12$, elongated wormlike micelles are suggested to be formed for short spacer lengths ( $s=2$ or 3 ), spherical micelles may be observed for spacer lengths in the range of 4-12, and for longer spacer lengths vesicles may be formed. ${ }^{26}$ However, aggregate structure also may be influenced by other factors, and in this study we anticipate that the two positively charged groups of each gemini may recognize neighboring negatively charged sulfonate groups on the polymer PBS-PFP, which could affect the structure of any micelles formed.

We report the interaction between the polyanion PBS-PFP and positively charged gemini surfactants of the $m-s-m$ type, with various spacer chain lengths for $m=12$ and with different lateral chain lengths for $s=2$. Absorption and emission spectroscopy and surface tension measurements are presented for a wide range of surfactant concentrations from the premicellar to postmicellar regions. In addition, molecular dynamics simulations have been carried out to provide an insight into the polymer-surfactant interactions in the premicellar region, while for one important case (12-6-12) SANS provide structural information on the aggregates formed and the influence of the polymer on the aggregate geometry at high surfactant concentrations (above the CMC). We believe this combination of techniques provides a coherent picture of the role of electrostatic and hydrophobic contributions to the polymer-surfactant interactions and to their effects on the polymer optical and photophysical properties and may help in the design of heterostructure devices containing conjugated polyelectrolytes.

\section{Experimental Section}

Materials. Poly \{1,4-phenylene-[9,9-bis(4-phenoxy-butylsulfonate)]fluorene-2,7-diyl\}copolymer, (PBS-PFP, $M_{n}$ 6500, Figure 1) was synthesized by condensation of 2,7-dibromo-9,9bis(4-sulfonylbutoxyphenyl)fluorene and 1,4-phenylenediboronic acid using $\mathrm{Pd}\left(\mathrm{PPh}_{3}\right)_{4}$ as a catalyst. ${ }^{22,23}$ Gemini surfactants $\alpha, \omega$ $\left(\mathrm{C}_{m} \mathrm{H}_{2 m+1} \mathrm{~N}^{+}\left(\mathrm{CH}_{3}\right)_{2}\right)_{2}\left(\mathrm{CH}_{2}\right)_{s}\left(\mathrm{Br}^{-}\right)_{2}$ were prepared according to the method described by Menger et al. ${ }^{29}$ Two sets of surfactants were used for the photophysical studies. The first group has 
dodecyl tail chains and spacers with 2, 3, 5, 6, and 12 carbon atom alkyl chains and the second one with 2 carbon atom spacer chains and tail chains with 12,16 , and 18 carbon atoms.

For the photophysical studies, freshly prepared PBS-PFP solutions with concentrations around $7 \times 10^{-3} \mathrm{~g} / \mathrm{L}$ (corresponding to $\sim 9.5 \times 10^{-6}$ (moles monomer)/L) were freshly prepared and were stirred overnight before use. Microliter additions of four surfactant stock solutions with concentrations around $10^{-4}$, $10^{-3}, 10^{-2}$, and $10^{-1} \mathrm{M}$ were made to provide a range of surfactant concentrations between $3 \times 10^{-8}$ and at least ca. 2.5 $\times 10^{-3} \mathrm{M}$, except for the poorly soluble $18-2-18$, where the maximum concentration used was $7.1 \times 10^{-4}$ M. Milli-Q Millipore water was used to prepare solutions.

For the surface tension measurements, small aliquots of gemini surfactant solution were sequentially added to a polymercontaining solution (kept at $0.21 \mathrm{mg} / \mathrm{mL}$ ), with dilution effects for the polymer taken into account. The surfactant concentration was varied roughly in the range of $10^{-5}-10^{-3} \mathrm{M}$.

For SANS measurements, samples were prepared by combining $\mathrm{D}_{2} \mathrm{O}$ (Goss Scientific Instruments, $99.9 \% \mathrm{D}$ minimum) mixtures of PBS-PFP (0.1 g/L) and 12-6-12 gemini surfactant $\left(1 \mathrm{~g} / \mathrm{L}, 1.5 \times 10^{-3} \mathrm{M}\right)$. Stirring for 1 day and ultrasound agitation for $10 \mathrm{~min}$ were used to ensure uniformity of the solutions. To avoid any degradation, fresh surfactant was used, and samples were measured within a few days of preparation.

Apparatus and Methods. Spectroscopic Measurements. Absorption spectral measurements were made in $1 \mathrm{~cm}$ quartz cuvettes on a Shimadzu UV-2100 spectrophotometer. The steady-state fluorescence spectra were measured with a HoribaJobin-Yvon-SPEX Fluorolog 3-22 instrument with $0.5 \mathrm{~nm}$ excitation and $0.5 \mathrm{~nm}$ emission bandwidths $\left(\lambda_{\mathrm{exc}}=381 \mathrm{~nm}\right)$. Fluorescence decays were measured using a home-built timecorrelated single photon counting apparatus consisting of an IBH NanoLED $\left(\lambda_{\text {exc }}=373 \mathrm{~nm}\right)$ as an excitation source, a JobinIvon monochromator, a Philips XP2020Q photomultiplier, and a Canberra Instruments time-to-amplitude converter and multichannel analyzer. Alternate measurements (1000 counts per cycle), controlled by Decay software (Biodinâmica-Portugal) of the pulse profile at $373 \mathrm{~nm}$ and the sample emission, were performed until $1-2 \times 10^{4}$ counts at the maximum were reached. ${ }^{30}$ The fluorescence decays were analyzed using the modulating functions method of Striker with automatic correction for the photomultiplier "wavelength shift". ${ }^{31}$ Although the lifetimes measured are at the limits of time resolution of our system, reproducible trends of the surfactant on the decays were observed. All experiments were carried out at room temperature (293 K).

Surface Tension Measurements. The surface tension measurements were performed with a software-controlled DCAT 11 tensiometer, from Dataphysics, with the Du Nöuy ring method. The temperature of the sample vessel was maintained with a thermostat bath at $25 \pm 0.1{ }^{\circ} \mathrm{C}$.

Simulations. Simulations were performed using the GROMACS software package with the standard GROMACS force field, ${ }^{32,33}$ which is a modified version of the GROMOS87 force field. ${ }^{34}$ Topology files were generated from initial structures, in Cartesian coordinates, resorting to the PRODRG server. ${ }^{35}$ The polymer and gemini surfactants were added to a box and solvated with single point charge (SPC) model water, ${ }^{36}$ with their structure constrained by the SETTLE algorithm. ${ }^{37}$ Five different simulations were conducted as summarized in Table 3 . These are compared with data focusing on the free polymer in water. To keep the whole system neutral different ions $\left(\mathrm{Na}^{+}\right.$, $\mathrm{Br}^{-}$) were added by randomly replacing solvent molecules.
TABLE 1: Lifetime Values of PBS-PFP Fluorescence in Aqueous Solution Alone and with Gemini Surfactants at Concentrations above Their CMCs

\begin{tabular}{cccc}
\hline surfactant & $\begin{array}{c}\text { PBS-PFP } \\
(\mathrm{g} / \mathrm{L})\end{array}$ & $\begin{array}{c}\text { surfactant concentration } \\
(\mathrm{mol} / \mathrm{L})\end{array}$ & $\begin{array}{c}\text { lifetime } \\
(\mathrm{ns})\end{array}$ \\
\hline none & $6.0 \times 10^{-3}$ & none & $0.30^{a}$ \\
$12-2-12$ & $7.2 \times 10^{-3}$ & $1.60 \times 10^{-3}$ & 0.48 \\
$12-5-12$ & $8.4 \times 10^{-3}$ & $1.85 \times 10^{-2}$ & 0.58 \\
$12-6-12$ & $8.4 \times 10^{-3}$ & $2.50 \times 10^{-2}$ & 0.57 \\
$16-2-16$ & $7.2 \times 10^{-3}$ & $7.70 \times 10^{-4}$ & 0.43 \\
$18-2-18$ & $7.2 \times 10^{-3}$ & $1.08 \times 10^{-3}$ & 0.40
\end{tabular}

${ }^{a}$ Data from ref 22

TABLE 2: CMCs and Interfacial Areas Per Molecule, $a$, for the Gemini and Mixed Gemini-Polymer Systems

\begin{tabular}{lcc}
\hline \multicolumn{1}{c}{ system } & $\begin{array}{c}\text { CMC } \\
(\mathrm{mM})\end{array}$ & $\begin{array}{c}a \\
\left(\mathrm{~nm}^{2}\right)\end{array}$ \\
\hline $12-2-12$ & $0.84 \pm 0.01$ & $1.1 \pm 0.1$ \\
$12-2-12+$ polymer & $0.84 \pm 0.01$ & $1.1 \pm 0.1$ \\
$12-6-12$ & $0.92 \pm 0.01$ & $1.3 \pm 0.1$ \\
$12-6-12+$ polymer & $0.92 \pm 0.01$ & $1.3 \pm 0.1$ \\
$12-10-12$ & $0.40 \pm 0.01$ & $1.5 \pm 0.1$ \\
$12-10-12+$ polymer & $0.39 \pm 0.01$ & $2.0 \pm 0.1$ \\
$12-12-12$ & $0.24 \pm 0.01$ & $2.0 \pm 0.1$ \\
$12-12-12+$ polymer & $0.21 \pm 0.01$ & $2.9 \pm 0.1$
\end{tabular}

TABLE 3: Components in Each Model System ${ }^{a}$

\begin{tabular}{lcccc}
\hline \multicolumn{1}{c}{ system } & $\begin{array}{c}\text { model oligomer } \\
\text { (4 repeat units) }\end{array}$ & gemini surfactant & water & counterions \\
\hline free polymer & 1 & & 6351 & $8 \times \mathrm{Na}^{+}$ \\
$12-2-12$ & 1 & $6 \times 12-2-12$ & 6487 & $4 \times \mathrm{Br}^{-}$ \\
$12-5-12$ & 1 & $6 \times 12-5-12$ & 6478 & $4 \times \mathrm{Br}^{-}$ \\
$12-6-12$ & 1 & $6 \times 12-6-12$ & 6614 & $4 \times \mathrm{Br}^{-}$ \\
$12-12-12$ & 1 & $6 \times 12-12-12$ & 6475 & $4 \times \mathrm{Br}^{-}$
\end{tabular}

${ }^{a}$ Free polymer denotes one oligomer in water, without surfactant molecules. The remaining systems, which include only one oligomer and six gemini molecules, are denoted according to the abbreviation for the gemini surfactant.

Previous to each molecular dynamics (MD) simulation, an energy minimization is performed. This is followed by a MD equilibration run under position restraints for $5 \mathrm{~ns}$. An unrestrained MD run is then carried out for $1 \mathrm{~ns}$ as a further equilibration simulation. Finally, a MD trajectory with a total length of $5 \mathrm{~ns}$ is generated with a time step of $2 \mathrm{fs}$.

All simulations are performed with periodic boundary conditions, using the Berendsen coupling algorithm. ${ }^{38}$ For this, it is necessary to specify pressure $(P)$, temperature $(T)$, and the respective coupling times $\tau_{P}$ and $\tau_{T}$. Thus, constant pressure, temperature, and number of particles $(N)$; i.e., $N P T$ conditions are maintained. We have used $P=1 \mathrm{bar}, \tau_{P}=0.5 \mathrm{ps}, T=300$ $\mathrm{K}$, and $\tau_{T}=0.1 \mathrm{ps}$. The particle mesh Ewald method ${ }^{39}$ was used for computation of long-range electrostatic forces.

Small-Angle Neutron Scattering Experiments. SANS experiments were performed using the SANS-1 instrument at the GKSS research center in Geesthacht, Germany. ${ }^{40}$ Several sample-to-detector distances (from 0.7 to $9.7 \mathrm{~m}$ ) and a wavelength of $8.1 \AA$ were employed to cover the range of scattering vector $q$ from 0.005 to $0.3 \AA^{-1}$. The wavelength resolution of the SANS-1 is $10 \%$ (full width at half-maximum). The samples were filled in Hellma quartz cells of $5 \mathrm{~mm}$ path length and placed in a thermostated holder, kept at $20.0 \pm 0.5{ }^{\circ} \mathrm{C}$. The raw scattering patterns were corrected for sample transmission, room background, and sample cell scattering by conventional procedures. ${ }^{41}$ The two-dimensional isotropic scattering patterns were azimuthally averaged, converted to an absolute scale, and corrected for detector efficiency by dividing by the incoherent 


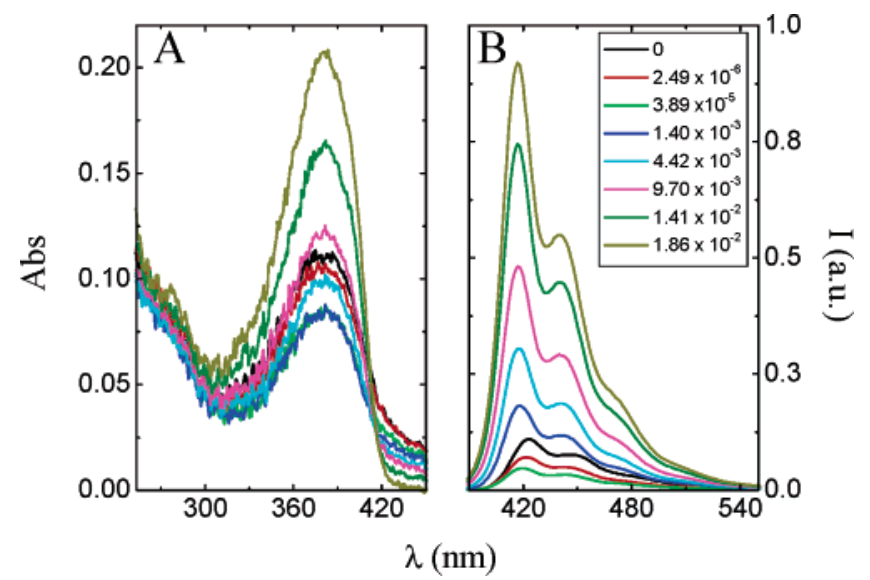

Figure 2. (A) Absorption and (B) emission spectra of PBS-PFP in aqueous solution $\left(8.4 \times 10^{-3} \mathrm{~g} / \mathrm{L}, 1.13 \times 10^{-5} \mathrm{~mol} / \mathrm{L}\right.$ in terms of repeat units) at various 12-5-12 molar concentrations. Note the apparent isosbestic point in part A around $410 \mathrm{~nm}$.

scattering spectra of 1-mm-thick pure water. The scattering from $\mathrm{D}_{2} \mathrm{O}$ used for the sample preparation was subtracted as a background; the small incoherent scattering due to the ${ }^{1} \mathrm{H}$ containing polymer was disregarded. The data for one sample were collected for $6 \mathrm{~h}$ on average. The absolute intensity SANS patterns were analyzed using the model-independent indirect Fourier transform (IFT) method developed by Glatter ${ }^{42}$ and using modeling of the interactive ellipsoids of revolution of Pedersen. ${ }^{43}$

\section{Results}

Photophysical and Spectroscopic Studies. Two sets gemini surfactants were used for the photophysical studies; the first had dodecyl tail chains changing the gemini surfactant spacer length $(2,3,5,6$, and 12 carbon atoms), while the second used 2 carbon atom spacers and different tail chain lengths $(12,16$, and 18 carbon atoms). The most marked effects came from the first set, where, although effects on the spectroscopic properties of PBS-PFP are qualitatively similar, there are important differences depending on the spacer chain length. Figure 2 illustrates the general behavior, with the absorption and emission spectra of PBS-PFP in aqueous solution following the addition of various concentrations of the gemini surfactant 12-5-12.

As can be seen in Figure 2A, the surfactant initially induces a decrease in the polymer absorption for concentrations below the surfactant $\mathrm{CMC}\left(\sim 1 \times 10^{-3} \mathrm{M}\right),{ }^{26}$ while increasing surfactant concentration leads to an increase in the absorbance of the polymer solution, a reduction in the light scattering, and a shift in the absorption maximum to the blue. Similar behavior is observed with other surfactants, although the observed shift depends upon the surfactant, with the largest shift (from 382 to $375 \mathrm{~nm}$ ) observed for 12-6-12. These modifications are accompanied by changes in the emission spectra, which are illustrated for 12-5-12 in Figure 2B. With all of the systems studied except for 12-12-12, the emission intensity is slightly quenched for surfactant additions below the $\mathrm{CMC}$ and increases dramatically above this concentration. These changes in emission intensity are accompanied by a slight blue shift in the emission maximum, a change in the vibronic structure, showing an increase in the intensity ratio of the main maximum to emission shoulder. A decrease (not shown) is also seen in the Rayleigh scattering peak. This contrasts with the behavior of the polymer in the presence of single-chain cationic tetraalkylammonium surfactants, ${ }^{24}$ where no change in spectral shape is seen in this quenching concentration range. For 12-12-12, the

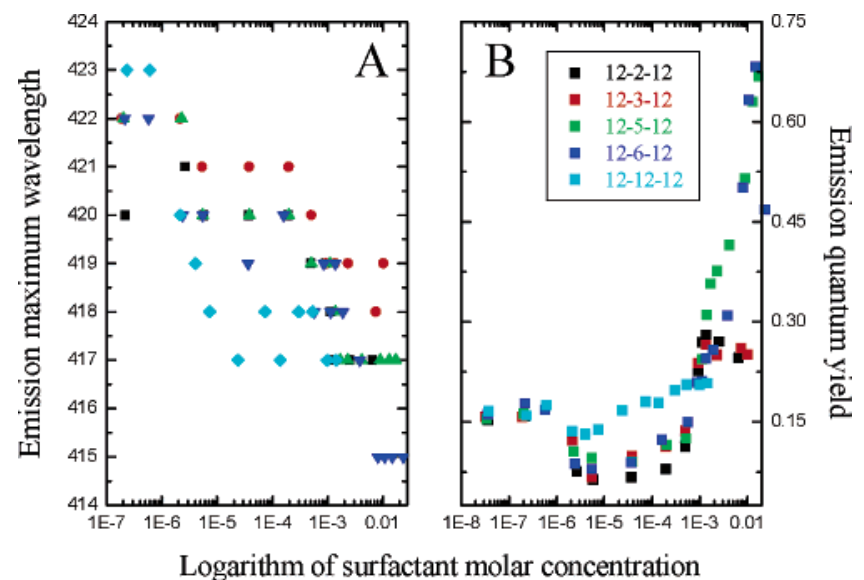

Figure 3. Shift of the emission maximum wavelength and changes in the emission quantum yield in the presence of different concentrations of gemini surfactants.

initial quenching is less important, and there is only a slight increase in the emission intensity for surfactant concentrations above $10^{-6} \mathrm{M}$ (well below the surfactant $\mathrm{CMC}$ ).

Although all of the gemini surfactants studied induced similar spectroscopic changes in PBS-PFP, there are significant differences between them as a function of the spacer chain length, as can be seen in Figure 3. The most dramatic increases in the emission quantum yield (Figure 3B) and the largest red shifts in the emission bands (Figure 3A) are induced by 12-512 and 12-6-12. A detailed analysis of Figure 3B shows that the polymer fluorescence quenching observed for surfactant concentrations below the $\mathrm{CMC}$ is most effective for gemini concentrations in the region of $5-10 \times 10^{-6} \mathrm{M}$, close to the polymer monomer concentration, and it seems probable that factors resulting from charge neutralization between gemini surfactant and PBS-PFP are responsible for the decrease in both the absorption and the emission intensities. Similar behavior was observed with the same polymer and single-chain cationic tetraalkylammonium surfactants, ${ }^{24}$ where the surfactant-neutralized polymer was suggested to form larger aggregates. However, in contrast to the behavior with these tetraalkylammonium surfactants, an increase in the absorption and emission intensities is observed for the gemini surfactants at concentrations above their CMC. This behavior has previously been observed upon addition of $n$-dodecyl pentaoxyethylene glycol ether $\left(\mathrm{C}_{12} \mathrm{E}_{5}\right)^{22}$ and other nonionic surfactants at concentrations above their CMCs to aqueous solutions of PBS-PFP. ${ }^{44}$ As with these cases, we believe that the dissolution of the polymer as isolated chains in surfactant micelles is responsible for the enhancement of the polymer spectroscopic properties. ${ }^{22}$

As was seen with $\mathrm{C}_{12} \mathrm{E}_{5},{ }^{22}$ gemini surfactants at concentrations above their CMC also increase the PBS-PFP fluorescence lifetime (Table 1). In agreement with the steady-state fluorescence data, the most marked effects on polymer fluorescent lifetimes are observed upon addition of 12-6-12 and 12-5-12 surfactants. The lifetimes in these cases are similar to those obtained upon the addition of $\mathrm{C}_{12} \mathrm{E}_{5}$ above its $\mathrm{CMC}$, which is compatible with dissolution of PBS-PFP as isolated chains, thereby reducing the efficiency of nonradiative decay pathways.

The structural reasons responsible for the pronounced effect of the 12-5-12 and 12-6-12 gemini surfactants on the photophysical properties will be considered in the Simulation subsection and the Discussion.

Spectroscopic and photophysical studies carried out on the polymer with gemini surfactants with 2 carbon atom spacers and variable tail chain lengths $(12,16$, and 18) show that these 

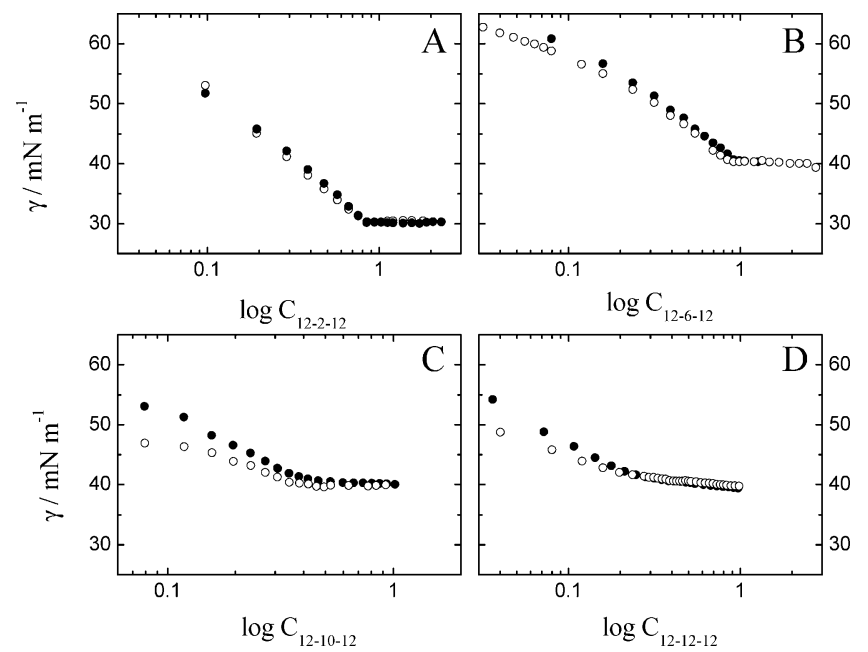

Figure 4. Surface tension vs $\log C$ plots for the gemini surfactant solutions without polymer (full circles) and in the presence of polymer (empty circles): (a) 12-2-12; (b) 12-6-12; (c) 12-10-12; (d) 12-12-12. The polymer concentration is constant at $0.21 \mathrm{mg} / \mathrm{mL}$.

induce similar effects to those of 12-2-12. However, the increase in the tail chain length leads to a dramatic increase in the light scattering, particularly for the 18 carbon atom system, probably due to the poor surfactant solubility. Since these do not lead to any improvements of the emission quantum yield, it would seem that the dodecyl surfactants are best suited for this study. In the next section we will address the effect of PBS-PFP on the surfactant properties of these gemini systems.

Surface Tension. Surface tension measurements were made on free surfactant solutions (12-2-12, 12-6-12, 12-10-12, and 12-12-12) and surfactant solutions with a polymer concentration of $\sim 0.21 \mathrm{mg} / \mathrm{mL}$. The plots of surface tension versus the surfactant concentration (mM) are shown in Figure 4. The CMCs for all of the systems together with the interfacial area per molecule, $a$, are presented in Table 2 .

The polymer alone has no effect on the surface tension of water, owing to its low aqueous solubility. From the experimental results shown in Figure 4 and Table 2, there appears to be practically no effect of the polymer on the CMC of the gemini surfactants, except for a very slight decrease observed with the longer spacers, 10 and 12. On the basis of the polymer molecular weight, the actual polymer (rather than repeat unit) concentration is $\sim 3.2 \times 10^{-5} \mathrm{M}$. Assuming micellar aggregation numbers for the gemini surfactants with $m=12$ of $\sim 45,{ }^{26}$ this means that the polymer and micellar concentrations are comparable for the surface tension measurements. This may suggest on the average incorporation of one polymer chain per micelle, without dramatic affects on micellar shape. Previous reports ${ }^{45}$ show that for surfactant concentrations above the CMC geminis with medium-sized spacer lengths form spheroidal micelles. Only 12-2-12 and 12-3-12 form elongated threadlike micelles but at concentrations markedly above the CMC, whereas for spacer length above 14 bilayer-like aggregates form in solution. Our SANS experiments with 12-6-12 in the absence and the presence of PBS-PFP confirm the formation of spherical aggregates that are not dramatically affected upon polymer addition (see SANS subsection).

In contrast with the lack of effect on the $\mathrm{CMC}$, from Figure 4 it can be seen that below the $\mathrm{CMC}$, for identical gemini concentrations, the surface tension is always lower in the presence of polymer than in the free gemini solution. This effect becomes more significant as the spacer length increases, being particularly evident for spacers 10 and 12. A likely explanation is that these surfactants complex with the polymer in the bulk liquid, as suggested from the photophysical measurements, leading to the breakup of PBS-PFP aggregates, while the resulting polymer-surfactant complex migrates and adsorbs at the interface. In other words, the surfactant is removing polymer from the bulk to the interface, producing a monolayer with lower surface tension than pure surfactant, with the effect being most marked for the more hydrophobic 12-10-12 and 12-12-12. Formation of polymer-surfactant monolayers, which can be transferred to appropriate substrates, has been confirmed with PBS-PFP and the nonionic $\mathrm{C}_{12} \mathrm{E}_{5}{ }^{17}$ and may be of value for device fabrication.

If we assume that the surface excess and interfacial area in the presence of polymer can be calculated through eqs 1 and 2 , respectively, as with solutions of the pure surfactant, then it can be seen that in the presence of PBS-PFP the maximum surface excess $\Gamma$ is lower for spacers 10 and 12 compared with the free gemini, indicating that the interfacial area per molecule, $a$, increases (Table 2)

$$
\begin{gathered}
\Gamma_{\max }=\frac{-1}{n R T}\left(\frac{\mathrm{d} \gamma}{\mathrm{d} \ln C}\right) \\
a=\frac{1}{\Gamma N_{\mathrm{A}}}
\end{gathered}
$$

In eq $1, n$ is considered equal to 3 , assuming complete dissociation of the surfactant and the two bromide counterions.

Surface tension data in the literature ${ }^{45}$ show that for this group of gemini surfactants, the average interfacial area per molecule, $a$, has a maximum around spacer lengths 10-12, implying that the charged part of the gemini surfactant containing the spacer interacts significantly with the aqueous side of the interface. The present results on the pure gemini systems are in complete agreement with this. Further, in the absence of polymer, the spacers 10 and 12 are already large enough to bend partially toward the air side of the interface, so despite the increase in $a$ on going from 12-6-12 to 12-10-12, the surfactants are probably not totally flat at the interface. When the polymer is adsorbed, the spacer and headgroups remain in contact with the aqueous phase, but due to interactions with PBS-PFP, having a rigid rod backbone, they are forced to into a more extended conformation at the interface. Hence, the area per molecule increases. The stronger effects on surface tension observed for spacers 10 and 12 can be explained by their higher hydrophobicity, which is confirmed by simulations (see next section). These show that Coulombic interactions are minimal for 1212-12 and that the hydrophobic interaction between this and the polymer shows no preference for the backbone or the alkyl chain.

Simulation Studies on PBS-PFP with Gemini Surfactants. To gain more insight into the $\mathrm{PBS}-\mathrm{PFP} / \mathrm{gemini}$ surfactant interactions, simulations have been performed on systems (Table 3) containing six gemini surfactant molecules per one PBSPFP model oligomer of four repeat units in a solvent box and give indications on how surfactant molecules interact with the polymer prior to aggregation into micelles. To obtain a rough idea of the Coulombic and hydrophobic interactions, several radial distribution functions of the charged and noncharged groups of both polymer and surfactants are presented as well as some snapshots of typical situations.

In Figure 5A, we depict the radial distribution function (RDF) for the sulfur atoms in the sulfonate group and the amine nitrogen atoms of the surfactants. It is clear that in the system 12-2-12 there is a close proximity between the charged groups 

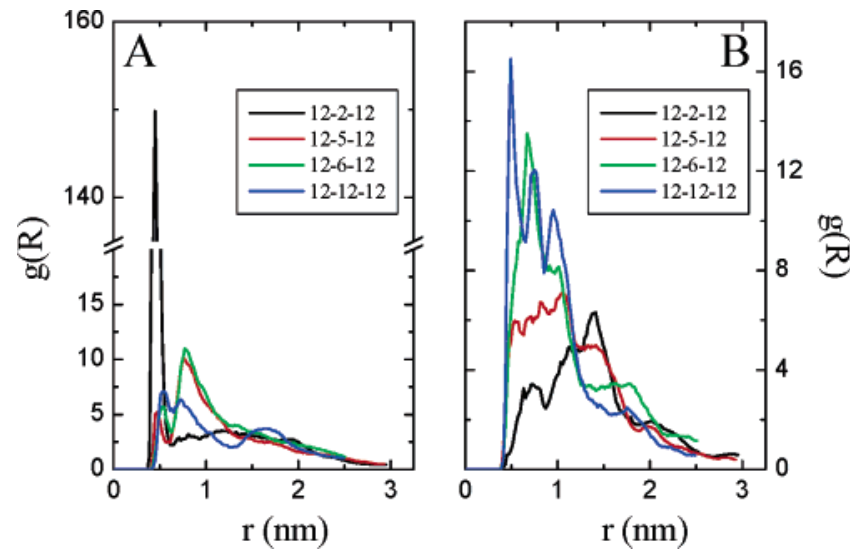

Figure 5. (A) Radial distribution functions for the polymer sulfur atoms and the surfactant nitrogen atoms for the different systems under study. (B) RDFs for central atoms in the fluorene group and the nitrogen atoms of the surfactant.

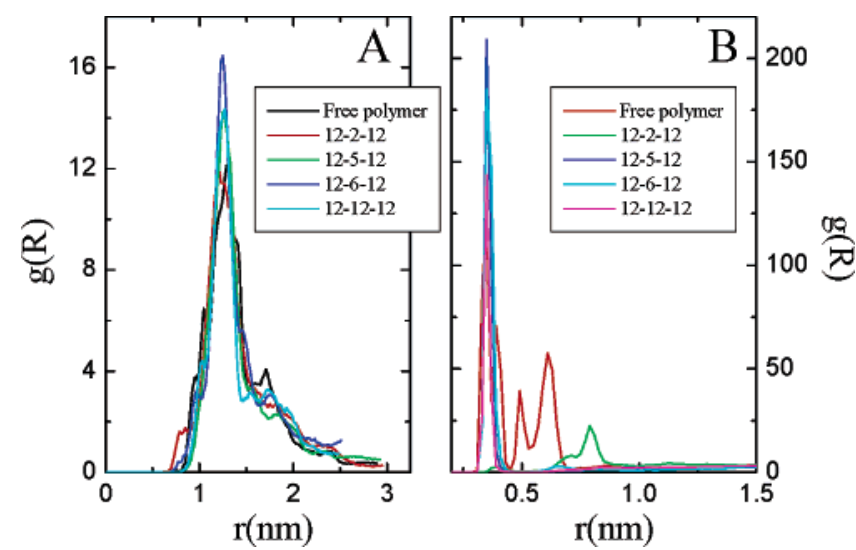

Figure 6. (A) RDFs for the central atoms in the fluorene groups and the sulfur atoms in the lateral chains and (B) the sulfur-sulfur RDF.

in both the oligomer and the surfactant. This preferential positioning becomes hardly noticeable as the spacer length increases. In contrast, the proximity between the amine groups in the gemini surfactant and the polymer backbone clearly increases, as can be seen in the RDF of Figure 5B. While the amine groups in system 12-2-12 are consistently situated far from the backbone, represented by the fluorene groups, with the respective RDF peaking at ca. $1.4 \mathrm{~nm}$, this value decreases to $1.1,0.7$, and $0.5 \mathrm{~nm}$ for systems $12-5-12,12-6-12$, and $12-$ 12-12, respectively. (Similar observations are made irrespective of the selection of atoms located in the backbone.) While the mechanisms for the initial fluorescence quenching and the decrease in absorbance seen in Figures 2 and 3 are not yet clear, the results show that both these result from charge neutralization. It is possible that this may be associated with the bromide counterions of the gemini surfactant, and these RDFs give some hints of possible differences between the various spacer lengths.

The behavior of the phenoxy-butylsulfonate chains in the polymer can be interpreted through Figure 6 . The degree of extension of these lateral chains from the backbone remains essentially unchanged upon addition of the surfactant (Figure $6 \mathrm{~A})$. However, the relative positioning of the sulfonate groups is affected, especially for the system 12-2-12, as seen in Figure $6 \mathrm{~B}$. Results on single PBS-PFP oligomers, with $\mathrm{Na}^{+}$counterions, indicate a well-defined relative positioning of the sulfonate groups. In fact, this figure presents four relatively sharp peaks centered at distances of $0.35,0.39,0.49$, and $0.61 \mathrm{~nm}$. The first peak corresponds to the closest sulfur atom, which is usually present in one adjacent monomer. The second peak is more
TABLE 4: Overall Conformational Indicators for the Oligomer Chain ${ }^{a}$

\begin{tabular}{lcc}
\hline \multicolumn{1}{c}{ system } & $\begin{array}{c}\text { average end-to-end } \\
\text { distance }(\mathrm{nm})\end{array}$ & $\begin{array}{c}\text { average radius } \\
\text { of gyration }(\mathrm{nm})\end{array}$ \\
\hline free model oligomer & 3.66 & 1.26 \\
$12-2-12$ & 3.68 & 1.30 \\
$12-5-12$ & 3.55 & 1.39 \\
$12-6-12$ & 1.88 & 1.39 \\
$12-12-12$ & 2.07 & 1.40
\end{tabular}

${ }^{a}$ The average end-to-end distance refers to the backbone (extreme carbon atoms), while the radius of gyration includes all groups. Errors, measured as one standard deviation, do not exceed $9 \times 10^{-3}$ and $5 \times$ $10^{-4} \mathrm{~nm}$, respectively.
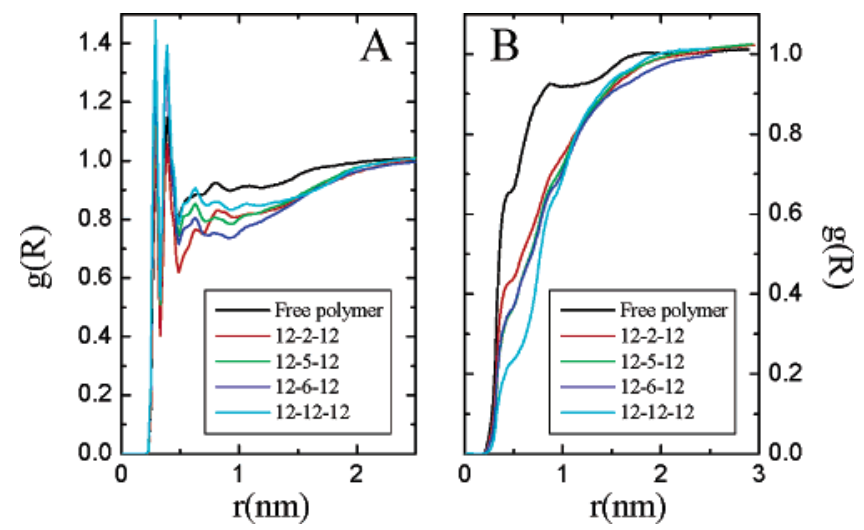

Figure 7. (A) Sulfur-water RDF and (B) fluorene-water RDF.

difficult to assign but may result partially from groups on the same monomer. However, when the polymer chain interacts with the surfactant molecules, the oligomer lateral chain positioning is substantially altered, and only one peak is observed in the RDF corresponding to systems $12-5-12,12-6-12$, and 12-1212. For system 12-2-12, the closest groups have a larger separation than in any of the other systems considered in this study.

The overall conformation of the oligomer is also affected by the presence of the surfactant molecules, as extracted from the respective values for the average end-to-end distance and the average radius of gyration (Table 4). In system 12-2-12, the oligomer end-to-end distance is very similar to that found for free polymer. For systems 12-5-12, 12-6-12, and 12-12-12, there is a general decrease in this distance, which is most noticeable for system 12-6-12. This means that the polyfluorene backbone shows its highest curvature when it interacts with 12-6-12. This is likely to help to disrupt polymer aggregates as the increase in the polyfluorene backbone curvature will make any $\pi-\pi$ interactions between the backbones of different polymer chains more difficult and simultaneously facilitate the incorporation of the polymer into micellar aggregates formed at the CMC. The presence of surfactant produces an increase in the radius of gyration of the polymer. This effect is largest for the larger spacer lengths and is minimal (ca. $0.04 \mathrm{~nm}$ ) for the 12-2-12 system.

In Figure 7, we represent the sulfur atom-water and the fluorene-water RDFs. The access to the solvent to the polymer charged groups (Figure 7A) is left almost unchanged in the presence of the 12-2-12 gemini surfactant. However, for those with a longer spacer, access is increased. If we now look at the fluorene-water RDF, Figure 7B, then it is seen that water has generally low access to the backbone, irrespective of the system. This access decreases still further in the presence of surfactants, with the effect being more pronounced as the spacer length increases. 

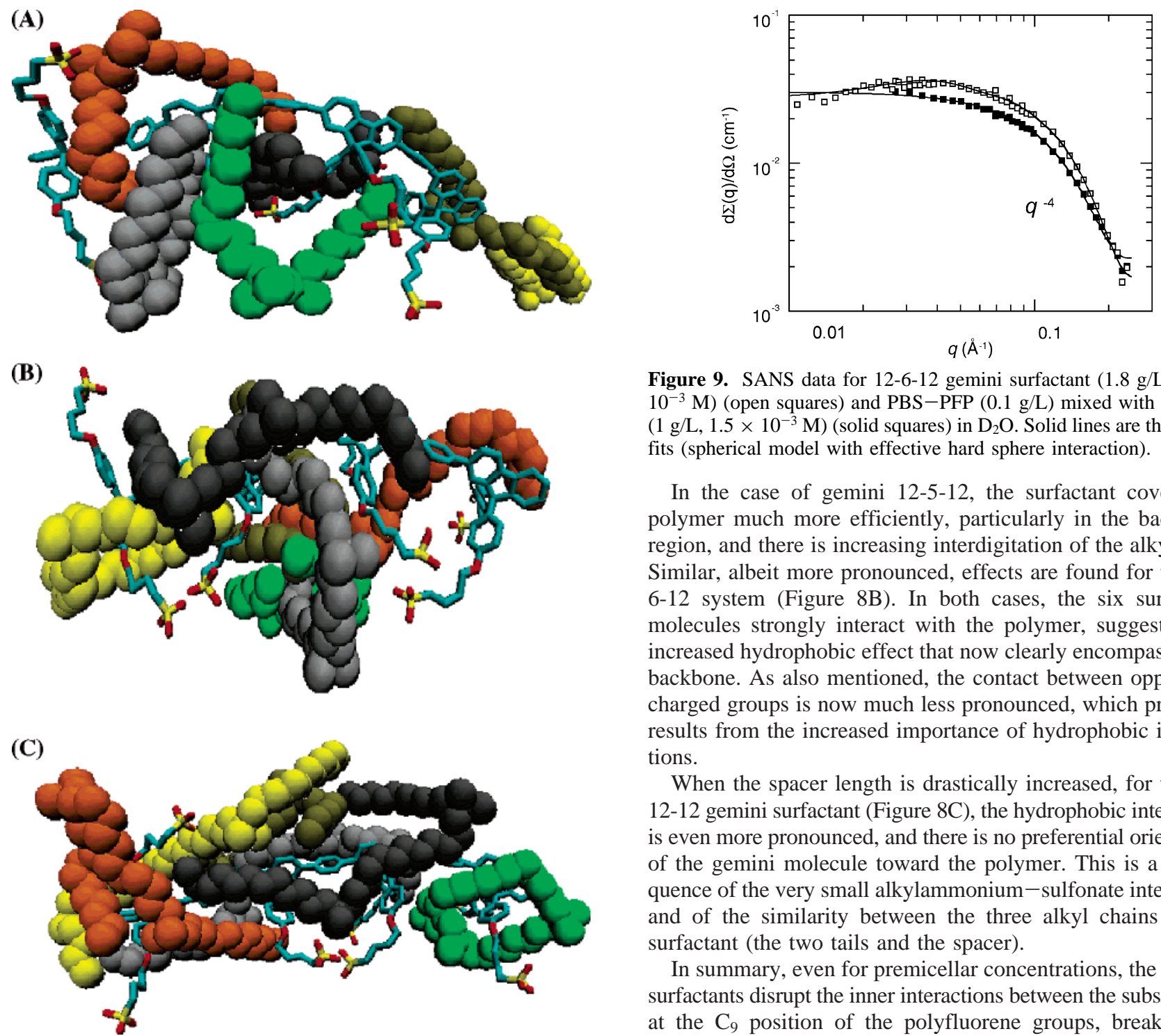

Figure 8. (A) Snapshot of system 12-2-12. Each gemini surfactant 12-2-12 is represented by a different color. Surfactant molecules are shown using the van der Waals radii for the respective atoms (space filling), while, for clarity, the oligomer is depicted in "sticks". The same for (B) 12-6-12 and (C) 12-12-12.

In general, it is apparent that the electrostatic interaction between the positively charged alkylammonium nitrogens and the oppositely charged sulfonates that favors the positioning of the 12-2-12 gemini becomes less and less important as the spacer length increases. At the same time, a closer proximity of the geminis to the backbone arises as a consequence of enhanced hydrophobic interaction, resulting in an additional depletion of water in this region of the oligomer.

The above observations can be illustrated by snapshots of the MD trajectory and are clear when the latter is observed as an animation. For the 12-2-12 surfactant, detailed observations of sequences of snapshots indicate that all six surfactant molecules in the simulation are in direct contact with the polymer. The alkylammonium groups are close to the sulfonate groups, as mentioned above, and the gemini alkyl chains directed toward the polymer backbone. The gemini act as tweezers that embrace the phenoxy-butylsulfonate chains (Figure 8A). These snapshots also confirm that direct contact between the gemini surfactant, especially the charged alkylammonium group, and the backbone is not frequent.

Figure 9. SANS data for 12-6-12 gemini surfactant $(1.8 \mathrm{~g} / \mathrm{L}, 2.7 \times$ $\left.10^{-3} \mathrm{M}\right)$ (open squares) and PBS-PFP $(0.1 \mathrm{~g} / \mathrm{L})$ mixed with $12-6-12$ $\left(1 \mathrm{~g} / \mathrm{L}, 1.5 \times 10^{-3} \mathrm{M}\right)$ (solid squares) in $\mathrm{D}_{2} \mathrm{O}$. Solid lines are the model fits (spherical model with effective hard sphere interaction).

In the case of gemini 12-5-12, the surfactant covers the polymer much more efficiently, particularly in the backbone region, and there is increasing interdigitation of the alkyl tails. Similar, albeit more pronounced, effects are found for the 126-12 system (Figure 8B). In both cases, the six surfactant molecules strongly interact with the polymer, suggesting an increased hydrophobic effect that now clearly encompasses the backbone. As also mentioned, the contact between oppositely charged groups is now much less pronounced, which probably results from the increased importance of hydrophobic interactions.

When the spacer length is drastically increased, for the 1212-12 gemini surfactant (Figure 8C), the hydrophobic interaction is even more pronounced, and there is no preferential orientation of the gemini molecule toward the polymer. This is a consequence of the very small alkylammonium-sulfonate interaction and of the similarity between the three alkyl chains in the surfactant (the two tails and the spacer).

In summary, even for premicellar concentrations, the gemini surfactants disrupt the inner interactions between the substituents at the $\mathrm{C}_{9}$ position of the polyfluorene groups, breaking up polymer aggregates and leading to a more expanded structure as taken from the radius of gyration of the oligomer. Also, they affect the overall configuration of the polymer and the corresponding interaction with the solvent and any counterions.

Small-Angle Neutron Scattering. Figure 9 plots the scattering curves of 12-6-12 gemini surfactant $\left(1.8 \mathrm{~g} / \mathrm{L}, 2.7 \times 10^{-3}\right.$ $\mathrm{M})$ in $\mathrm{D}_{2} \mathrm{O}$ and PBS-PFP $(0.1 \mathrm{~g} / \mathrm{L})$ mixed with $12-6-12(1 \mathrm{~g} / \mathrm{L}$, $1.5 \times 10^{-3} \mathrm{M}$ ) in the same solvent as well as model fits (sphere with a hard sphere interaction). Figure 10 plots corresponding pair distance distribution functions calculated using the IFT method. Both curves in Figure 9 show a nearly plateau region at lower $q$ and a characteristic Porod law scattering at higher $q$, potentially arising from the small three-dimensional (spherical) particles. Also the symmetrical shape of the correlation functions shown in Figure 10 supports the idea of the spherical shape of the aggregates.

Inspecting Figure 9 further, the SANS curve of the gemini surfactant $/ \mathrm{D}_{2} \mathrm{O}$ system shows an additional broad interference maximum at $q \approx 0.04 \AA^{-1}$ that can be attributed to the electrostatic repulsion between aggregates. This effect of interaction on scattering curve should be negligible for the $q$ range greater than $0.05 \AA^{-1}$, and therefore the IFT was applied only for this interval. The IFT analysis gives the radius of gyration $1.35 \pm 0.03 \mathrm{~nm}$ corresponding to a homogeneous sphere with the radius of $1.74 \pm 0.04 \mathrm{~nm}$. The interaction between aggregates was also taken into account by fitting the 


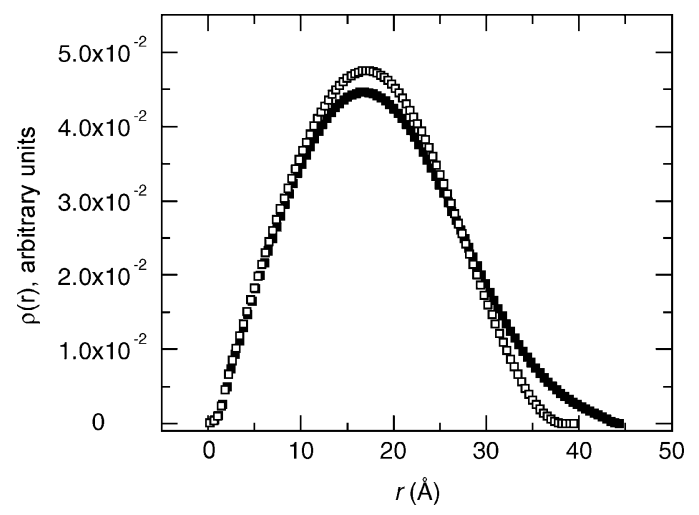

Figure 10. Pair distance distribution function calculated from the SANS data for 12-6-12 gemini surfactant $\left(1.8 \mathrm{~g} / \mathrm{L}, 2.7 \times 10^{-3} \mathrm{M}\right)$ (open squares) and PBS-PFP (0.1 g/L) mixed with 12-6-12 (1 g/L, $1.5 \times$ $10^{-3} \mathrm{M}$ ) (solid squares) shown in Figure 9.

data to a model of effective hard sphere interaction. This means that the radius of a hard sphere interaction is larger than that of a particle with electrostatic interaction. The fitting by the ellipsoid of revolution shows that aggregates are spherical (axis ratio is equal to 1 within statistical error) with a radius of 1.77 $\pm 0.03 \mathrm{~nm}$. To obtain a reasonable fit to the hard sphere, a radius of $6.5 \mathrm{~nm}$ is required.

The SANS curve of the $\mathrm{D}_{2} \mathrm{O}$ solution of $12-6-12$ with the polymer (Figure 9) has no interference maximum, which may indicate that the anionic polymer screens (at least partially) the charges of the cationic surfactant. This may mean that the polymer is incorporated into the surfactant aggregates or that the polymer is attached to the surface of the surfactant aggregates. If the polymer is to some extent incorporated into the surfactant aggregates, then a small increase in the size of the aggregates should be observed. Both analyses of the data of the aqueous PBS-PFP/gemini surfactant system indicate the formation of small spherical objects. The IFT method gives the radius of gyration of $1.44 \pm 0.03 \mathrm{~nm}$, which corresponds to a homogeneous sphere with a radius of $1.86 \pm 0.04 \mathrm{~nm}$. Modeling by ellipsoid of revolution suggests spherical aggregates (axis ratio is equal to 1 within statistical error) with a radius of 1.86 $\pm 0.02 \mathrm{~nm}$. As the difference in the particle size is small, the results do not allow unambiguous identification as to which of these two possible modes of polymer association with surfactant micelles is occurring. In addition, the SANS method does not allow studies at the low concentrations employed in the spectroscopic measurements, such that any comparison between scattering and photophysical results remains qualitative. However, we can safely conclude that the simple changes observed in the SANS data on mixing the constituents are in phenomenological agreement with the formation of $\mathrm{PBS}-\mathrm{PFP} / \mathrm{gemini}$ surfactant aggregates.

\section{Discussion}

The interaction between gemini surfactants, $m-s-m$, and PBSPFP in aqueous solutions modifies the optical and photophysical properties of the polymer, and shows a complex dependence on the surfactant concentration and spacer chain length. In addition to any geometric factors associated with the relation between the surfactant spacer length and the intercharge separation on the polymer, the results imply a balance between different interactions, notably Coulombic attraction between surfactant and polymer and hydrophobic effects. In all cases, upon adding surfactant there is an initial marked decrease in the polymer absorbance, which is followed at higher surfactant concentrations by an increase in absorbance, a blue shift in the maximum, and a sharpening of the band shape. In this concentration range, changes also occur in the fluorescence spectra, which in all cases except 12-12-12 show a quenching in the region of neutralization of the polymer charge by the surfactant, accompanied by a blue shift in emission maxima. We have previously shown on interaction of this polymer with the nonionic $\mathrm{C}_{12} \mathrm{E}_{5}$ that this blue shift is very similar to the spectral change observed with polyfluorenes on going from thin films to dilute solution ${ }^{22}$ and can be explained by breakup of polymer aggregates. SANS and NMR studies support this. ${ }^{17,23}$ Although the initial fluorescence quenching induced by the gemini surfactants is associated with charge neutralization, the actual mechanism is still unclear. However, it is known that both water ${ }^{46}$ and bromide ions ${ }^{47}$ are quenchers of aromatic fluorescence. From the molecular simulations, the fluorenewater RDFs in Figure 7B show that there may be some penetration of the water (and presumably counterions) to the polymer backbone for 12-2-12, with rather less for 12-5-12 and $12-6-12$ and very little in the case of 12-12-12. It is noteworthy that in the case of 12-12-12 little fluorescence quenching is seen in this concentration region. The simulations also show strong hydrophobic interactions between PBS-PFP and 12-12-12, which may explain the tendency of this system to adsorb at the interface as seen in surface tension measurements.

In the region of the surfactant CMC the fluorescence intensity increases, which may be associated with incorporation of single chains of the polymer in surfactant micelles. Surface tension measurements show that this occurs without significantly affecting the CMC. This is similar to what is observed with this polymer and the neutral alkyloxyethylene surfactants. ${ }^{22}$ However, somewhat different efficiencies of fluorescence enhancement are observed with different spacer lengths. These simulations indicate the complex interplay between Coulombic and hydrophobic effects, with 12-2-12 being dominated by the former and 12-12-12 by the latter. The intermediate 12-5-12 and 12-6-12 seem to strike a good hydrophile-lipophile balance, leading to the maximum enhancement of fluorescence. In fact, the fluorescence quantum yields at the highest surfactant concentrations in these cases are virtually identical to those seen with $\mathrm{PBS}-\mathrm{PFP}$ in $\mathrm{C}_{12} \mathrm{E}_{5}$ or Triton $\mathrm{X}-100$ solutions $^{22,44}$ and probably represent the natural quantum yield of isolated chains of this polymer in the absence of quenchers.

Scattering methods provide an excellent way of characterizing surfactant aggregates in solution. Unfortunately it is normally difficult to perform these studies in the same concentration range as photophysical and spectroscopic studies. With the surfactant 12-6-12, it was possible to obtain structural information on micellar aggregates in the absence and presence of PBS-PFP, albeit at higher polymer concentrations than with the photophysical studies. These show that the surfactant produces spherical micelles in the concentration range studied, in agreement with previous results, ${ }^{26}$ and that the presence of the polymer only slightly increases the size of these. The results are in agreement with the lack of effect of the polymer on the CMC values of this and the other gemini surfactants, as determined by surface tension.

\section{Conclusions}

Gemini surfactants, generally represented as $\mathrm{C}_{s} \mathrm{H}_{2 s}-\alpha, \omega$ $\left(\left(\mathrm{CH}_{3}\right)_{2} \mathrm{~N}^{+} \mathrm{C}_{m} \mathrm{H}_{2 m+1} \mathrm{Br}^{-}\right)_{2}(m-s-m)$ with 12 atoms in the lateral chain (12) and different spacer chain lengths $(s=2,5,6,10$, and 12) interact with PBS-PFP even at very low surfactant concentrations. Similar interactions occur with longer lateral 
chain surfactants, but very poor solubility limits their applications in these systems.

Because of its high hydrophobicity, as shown in simulations, 12-12-12 interacts at very low concentrations both with the polyfluorene and the alkyl chain, and induces a slight increase of the polymer emission intensity at surfactant concentrations at least an order of magnitude below the CMC. Associated with the high hydrophobicity, this long spacer chain surfactant also shows high surface activity, leading to adsorption at the airwater interface.

In contrast, at low surfactant concentrations, simulations of the interactions of PBS-PFP with gemini surfactants with very small spacer chain lengths, such as 12-2-12, show that the interaction is mainly electrostatic between the oppositely charged tetraalkylammonium group of the surfactant and the sulfonate of the polymer, which may be associated with the start of breakup of polymer aggregates, as indicated by blue shifts in fluorescence maxima. Upon neutralization of the polymer charge, slight quenching of its fluorescence is observed, while with surfactant concentrations around the $\mathrm{CMC}$, the polymer aggregates are completely disrupted and become incorporated into surfactant micelles, leading to increases in the polymer emission quantum yield. Qualitatively similar changes in fluorescence behavior at low surfactant concentrations but much larger fluorescence quantum yields above the CMC are found when PBS-PFP interacts with 12-5-15 and 12-6-12, which coincides with the optimum match between the spacer length of the surfactant and the intercharge separation between adjacent monomers on the polyelectrolyte. Simulations in these cases suggest a good balance between Coulombic and hydrophobic effects and show that the surfactant interacts mainly with the polyfluorene backbone even at very low concentrations. This leads to bending of the polymer backbone, which facilitates the disruption of polymer aggregates and probably makes the polymer interaction with surfactant aggregates easier. While these observations are of a qualitative nature, they do allow the design of new functional materials in which molecular recognition between oppositely charged conjugated polyelectrolytes and gemini surfactants leads to deaggregation of the polymer. Further studies are in progress to see the effect of changing the intercharge spacing on these two entities and obtain more quantitative information on the importance of the various factors discussed above.

Surface tension experiments show that presence of the polymer does not affect the surfactant CMC, while SANS studies with 12-6-12 show the formation of spherical aggregates and suggest a slight increase in radius from $1.74 \pm 0.04$ to 1.86 $\pm 0.04 \mathrm{~nm}$, compatible with PBS-PFP/micelle interactions.

Acknowledgment. SANS measurements have been supported by the European Commission under the sixth Framework Program through the Key Action: Strengthening the European Research Area, Research Infrastructures (Contract No. RII3CT-2003-505925). Preliminary SANS experiments were carried out at the ISIS Facility, RAL, Chilton, Didcot, U. K. We are indebted to Dr. S. M. King for his assistance with these. MEC and FEDER are thanked for financial support through Project No. MAT2004-03827 and POCTI/FCT/FEDER through Project No. POCTI/39593/QUI/2001. We are grateful for further funding for this collaboration through MEC/CRUP (Acções Integradas), Acción Integrada Hispano-Portuguesa (HP20030077), the Royal Society, and the European Union COST Action D15 WG 0017-00. S.M.F. and J.P. thank the FCT for postdoc- toral and Ph.D. grants (SFRH/BD/18876/2004). The Universidad de Burgos is also thanked for financial support of a short stay of M.J.T. at Coimbra University.

\section{References and Notes}

(1) Lehn, J.-M. Supramolecular Chemistry; VCH: Weinheim, Germany, 1995 .

(2) Decher, G. Science 1997, 277, 1232-1237. 7080 .

(3) Zhou, X.; Yan, J.; Pei, J. Macromolecules 2004, 37, 7078-

(4) Liu, B.; Bazan, G. C. Chem. Mater. 2004, 16, 4467-4476.

(5) Wosnick, J. H.; Mello, C. M.; Swager, T. M. J. Am. Chem. Soc. 2005, 127, 3400-3405.

(6) Chen, Y.; Araki, Y.; Doyle, J.; Strevens, A.; Ito, O.; Blau, W. J. Chem. Mater. 2005, 17, 1661-1666.

(7) Cho, N. S.; Hwang, D.; Jung, B.; Lim, E.; Lee, J.; Shim, H. Macromolecules 2004, 37, 5265-5273.

(8) Ego, C.; Marsitzky, D.; Becker, S.; Zhang, J.; Grimsdale, A. C.; Müllen, K.; Mackenzie, J. D.; Silva, C.; Friend, R. H. J. Am. Chem. Soc. 2003, 125, 437-443.

(9) Arias, A. C.; Mackenzie, J. D.; Stevenson, R.; Halls, J. J. M.; Inbasekaran, M.; Woo, E. P.; Richards, D.; Friend, R. H. Macromolecules 2001, 34, 6005-6013.

(10) Grell, M; Bradley, D. D. C.; Long, X.; Chamberlain, T.; Inbasekaran, M.; Woo, E. P.; Soliman, M. Acta Polym. 1998, 49, 439-444.

(11) Grell, M; Bradley, D. D. C.; Ungar, G.; Hill, J.; Whitehead, K. S. Macromolecules 1999, 32, 5810-5817.

(12) Menon, A.; Galvin, M.; Walz, K. A.; Rothberg, L. Synth. Met. 2004, 141, 197-202.

(13) Wu, C.-W.; Tsai, C.-M.; Lin, H.-C. Macromolecules 2006, 39, $4298-4305$

(14) Xia, C.; Advincula, R. C. Macromolecules 2001, 34, 58545859 .

(15) Wang, D.; Moses, D.; Bazan, G. C.; Heeger, A. J.; Lal, J. J. Macromol. Sci., Pure Appl. Chem. 2001, 38, 1175-1189.

(16) Gaylord, B. S.; Wang, S.; Heeger, A. J.; Bazan, G. C. J. Am. Chem. Soc. 2001, 123, 6417-6418.

(17) Knaapila, M.; Almásy, L.; Garamus, V. M.; Pearson, C.; Pradhan, S.; Petty, M. C.; Scherf, U.; Burrows, H. D.; Monkman, A. P. J. Phys. Chem. B 2006, 110, 10248-10257.

(18) Nguyen, B. T.; Gautrot, J. E.; Ji, C.; Brunner, P. L.; Nguyen, M. T.; Zhu, X. X. Langmuir 2006, 22, 4799-4803.

(19) Abe, S.; Chen, L. J. Polym. Sci., Part B: Polym. Phys. 2003, 41, $1676-1679$

(20) Chen, L.; McBranch, D.; Wang, R.; Whitten, D. Chem. Phys. Lett. 2000, 330, 27-33.

(21) Chen, L.; Xu, S.; McBranch, D.; Whitten, D. J. Am. Chem. Soc. 2000, 122, 9302-9303.

(22) Burrows, H. D.; Lobo, V. M. M.; Pina, J.; Ramos, M. L.; Seixas de Melo, J.; Valente, A. J. M.; Tapia, M. J.; Pradhan, S.; Scherf, U. Macromolecules 2004, 37, 7425-7427.

(23) Burrows, H. D.; Lobo, V. M. M.; Pina, J.; Ramos, M. L.; Seixas de Melo, J.; Valente, A. J. M.; Tapia, M. J.; Pradhan, S.; Scherf, U.; Hintschich, S. I.; Rothe, C.; Monkman, A. P. Colloids Surf., A 2005, 270 $271,61-66$

(24) Tapia, M. J.; Burrows, H. D.; Valente, A. J. M.; Pradhan, S.; Scherf, U.; Lobo, V. M. M.; Pina, J.; Seixas de Melo, J. J. Phys. Chem. B 2005 , 109, 19108-19115

(25) Tapia, M. J.; Burrows, H. D.; Knaapila, M.; Monkman, A. P.; Arroyo, A.; Pradhan, S.; Scherf, U.; Pinazo, A.; Pérez, L.; Morán, C. Langmuir 2006, 22, 10170-10174.

(26) Hait, S. K.; Moulik, S. P. Curr. Sci. 2002, 82, 1101-1111.

(27) Zana, R.; Xia, J. In Gemini Surfactants: Synthesis, Interfacial and Solution Phase Behaviour, and Applications; Marcel Dekker: New York, 2003.

(28) Oda, R.; Huc, I.; Candau, S. J. Chem. Commun. 1997, 21052106.

(29) Menger, F. M.; Littau, C. A. J. Am. Chem. Soc. 1991, 113, 14511452

(30) Seixas de Melo, J.; Fernandes, P. F. J. Mol. Struct. 2002, 565, 6974.

(31) Striker, G.; Subramaniam, V.; Seidel, C. A. M.; Volkmer, A. J. Phys. Chem. B 1999, 103, 8612-8617.

(32) Berendsen, H. J. C.; van der Spoel, D.; van Drunen, R. Comput. Phys. Commun. 1995, 91, 43-56.

(33) Lindhal, E.; Hess, B.; van der Spoel, D. J. Mol. Graphics Modell. 2001, 7, 306-317.

(34) van Gunsteren, W. F.; Berendsen, H. J. C. Gromos-87 Manual; Biomos BV: Groningen, The Netherlands, 1987. 
(35) van Aalten, D. M. F.; Bywater, R.; Findlay, J. B. C.; Hendlich, M.; Hooft, R. W. W.; Vriend, G. J. Comput.-Aided Mol. Des. 1996, 10 , 255-263.

(36) Berendsen, H. J. C.; Postma, J. P. M.; van Gunsteren, W. F.; Hermans, J. SPC. In Intermolecular Forces; Pullman, B., Ed.; Reidel: Dordrecht, The Netherlands, 1981; pp 331-342.

(37) Miyamoto, S.; Kollman, P. A. J. Comput. Chem. 1992, 13, 952962.

(38) Berendsen, H. J. C.; Postma, J. P. M.; DiNola, A.; Haak, J. R. J. Chem. Phys. 1984, 81, 3684-3690.

(39) Darden, T.; York, D.; Pedersen, L. J. Chem. Phys. 1993, 98, 1008910092

(40) Stuhrmann, H. B.; Burkhardt, N.; Dietrich, G.; Junemann, R.; Meerwinck, W.; Schmitt, M.; Wadzack, J.; Willumeit, R.; Zhao, J.; Nierhaus,
K. H. Nucl. Instrum. Methods Phys. Res., Sect. A 1995, 356, 124132.

(41) Wignall, G. D.; Bates, F. S. J. Appl. Crystallogr. 1987, 20, $28-$ 40.

(42) Glatter, O. J. Appl. Crystallogr. 1977, 10, 415-421.

(43) Pedersen, J. S. Adv. Colloid Interface Sci. 1997, 70, 171210 .

(44) Burrows, H. D.; Tapia, M. J.; Fonseca, S. M.; Pradhan, S.; Scherf, U.; Silva, C. L.; Pais, A. A. C. C. J. Chem. Phys., submitted for publication.

(45) Zana, R. Adv. Colloid Interface Sci. 2002, 97, 205-253.

(46) Luria, M.; Stein, G. J. Phys. Chem. 1972, 76, 165-171.

(47) Watkins, A. R. J. Phys. Chem. 1974, 78, 2555-2558. 